\title{
Impacto das Consequências Psicossociais do Estigma do Peso no Tratamento da Obesidade: uma Revisão Integrativa da Literatura
}

\author{
Maraisa Tarozo ${ }^{1}$ \\ ${ }^{1}$ Universidade de São Paulo, SP, Brasil.
}

\author{
Rosane Pilot Pessa ${ }^{1}$ \\ ${ }^{1}$ Universidade de São Paulo, SP, Brasil.
}

\begin{abstract}
Resumo: Trata-se de uma revisão integrativa da literatura, cujo objetivo foi investigar as consequências psicossociais do estigma do peso em adultos e sua influência no tratamento da obesidade. A busca envolveu as bases de dados PubMed, Web of Science e PsycINFO e incluiu artigos em inglês e português publicados nos últimos cinco anos. Foram selecionados quinze artigos, analisados a partir da definição de dois subtemas: "A relação entre estigma, psicopatologias e transtornos do comportamento alimentar" e "A estigmatização entre os profissionais de saúde". Os resultados apontam para o grande impacto do estigma do peso na saúde psicossocial da pessoa com obesidade e para as atitudes estigmatizantes dos profissionais de saúde no cuidado terapêutico. Observa-se um enfoque patológico do enfrentamento da obesidade com estratégias de cunho comportamental que minimizam a compreensão da problemática. Atualmente, o tratamento da obesidade promovido pelo modelo biomédico não abrange as demandas de caráter psicossocial, o que torna necessário a educação permanente para capacitação dos profissionais de saúde e a implementação de protocolos de intervenção específicos a este grupo populacional. A contribuição de profissionais de diversas áreas, e em especial, daqueles de saúde mental, é essencial em atenção às diferentes particularidades do tratamento, devendo ser baseada em uma perspectiva integral e humanizada, e inserida nos diferentes contextos psicossociais. Devido à perversidade do estigma do peso e suas graves consequências, futuros estudos são necessários para investigação desse problema e das atitudes dos profissionais da saúde, familiares, mídia e da população em geral em relação à pessoa com obesidade.
\end{abstract}

Palavras-chave: Estigma, Obesidade, Consequências Psicossociais, Tratamento, Profissionais de Saúde.

\section{Impact of Psychosocial Consequences of Weight Stigma in the Treatment of Obesity: an Integrative Literature Review}

\begin{abstract}
This paper is an integrative review of the literature whose objective was to investigate the psychosocial consequences of weight stigma in adults and its influence on the treatment of obesity. The search involved the PubMed, Web of Science and PsycINFO databases and included articles in English and Portuguese published during the last five years. We selected 15 articles, analyzed according to the definition of two subtopics: "The relationship between stigma, psychopathologies and eating disorders" and "Stigmatization among health professionals". The results point to the great impact of weight stigma on the psychosocial health of obese individuals and the stigmatizing attitudes of health professionals in therapeutic care. A pathological approach to coping with obesity using behavioral strategies that minimizes the understanding of the problem was observed. Currently, the treatment of obesity promoted by the biomedical model does not include the demands of a psychosocial character, which makes permanent education necessary for training of health professionals and the implementation of specific intervention protocols for this population group. The contribution of professionals from different areas, and
\end{abstract}


especially those of mental health, is essential to pay attention to the various characteristics of the treatment, following an integral and humanized perspective, inserted in different psychosocial contexts. Due to the perversity of weight stigma and its serious consequences, further studies are needed to investigate it as well as the attitudes of health professionals, family members, the media and the general population towards obese individuals.

Keywords: Stigma, Obesity, Psychosocial Consequences, Treatment, Health Professionals.

\title{
Impacto de las Consecuencias Psicosociales del Estigma de Peso en el Tratamiento de la Obesidad: una Revisión Integrativa de la Literatura
}

\begin{abstract}
Resumen: Se trata de una revisión integrativa de la literatura, cuyo objetivo fue investigar la consecuencias psicosociales del estigma del peso en los adultos y su influencia en el tratamiento de obesidad. Se realizó una búsqueda en las bases de datos PubMed, Web of Science y PsycINFO de artículos en inglés y portugués publicados en los últimos cinco años. Se seleccionaron 15 artículos para análisis a partir de la definición de dos subtemas: "la relación entre el estigma, psicopatologías y trastornos del comportamiento alimenticio" y "la estigmatización entre profesionales de la salud". Los resultados apuntan al gran impacto del estigma de peso en la salud psicosocial de la persona con obesidad y las actitudes estigmatizantes de los profesionales de la salud en el cuidado terapéutico. Se observa un abordaje patológico de confrontación de la obesidad con estrategias de comportamiento que minimizan la comprensión del problema. Actualmente, el tratamiento de la obesidad promovido por el modelo biomédico no cubre las exigencias de un carácter psicosocial, que hace necesaria la educación continua para capacitar a los profesionales de la salud e implementar protocolos de intervención específicos a ese grupo poblacional. La contribución de profesionales de diferentes áreas, especialmente los de la salud mental, es esencial en la atención a las diferentes particularidades del tratamiento, que debe basarse en una perspectiva integral y humanizada e insertarse en los diferentes contextos psicosociales. Debido a la perversidad del estigma de peso y sus graves consecuencias, se necesitan estudios futuros para investigar el estigma del peso y las actitudes de los profesionales de la salud, la familia, los medios de comunicación y la población en general en relación a la persona con obesidad.
\end{abstract}

Palabras clave: Estigma, Obesidad, Consecuencias Psicosociales, Tratamiento, Profesionales de la Salud.

\section{Introdução}

$\mathrm{O}$ estigma relacionado à obesidade retrata um conceito social cuja aplicação resulta em graves danos, de ordem física e psicossocial, àqueles que possuem excesso de peso (Wu \& Berry, 2017). Segundo Goffman (1988), o estigma representa uma "marca" ou "atributo" para discriminar, afastar ou excluir o indivíduo que possui características discrepantes daquelas consideradas adequadas por uma sociedade. A estigmatização ocorre a partir da observação de características consideradas inadequadas e atribuição de estereótipos negativos aos portadores (Goffman, 1988; Major \& O’Brien, 2005).

Nesse contexto, o excesso de tecido adiposo é considerado uma condição indesejada, inadequada e divergente do padrão corporal socialmente estabelecido pela cultura ocidental, que enaltece o corpo magro e desqualifica o corpo da pessoa com obesidade (Puhl, Quinn, Weisz, \& Suh, 2017). O corpo volumoso é visto como sinônimo de incompetência, desleixo e falta de controle nos diversos contextos sociais, enquanto o corpo magro encontra-se associado à 
disciplina, força de vontade e constância de hábitos (Pearl \& Lebowitz, 2014; Puhl \& Suh, 2015).

O estigma do peso ocorre diante da associação entre excesso de peso e o insucesso em seguir hábitos de vida saudáveis, e desse modo atribui ao indivíduo atingido culpa por sua condição, sem considerar outras possíveis causas (Gracia Arnaiz, 2010). As preconcepções sobre os fatores etiológicos da obesidade culminam em discriminação, visto que o excesso de tecido adiposo configura-se como um atributo desviante que é alvo de estereótipos negativos, ou seja, de estigmatização (Brewis, Wutich, Falletta-Cowden, \& Rodriguez-Soto, 2011).

Indivíduos com obesidade apresentam, no ambiente de trabalho, desvantagens na contratação, salários e promoções em consequência de seu peso; no meio educacional, são estigmatizados por professores e colegas. Diante da mídia, aos personagens magros são atribuídos características desejáveis e papéis importantes, enquanto as pessoas com obesidade são frequentemente observadas em papéis estereotipados. Nesse sentido, ocorre uma explicitação da aceitabilidade social do estigma de peso, em que a pessoa com obesidade é alvo de depreciação. Em relações interpessoais são observadas atitudes estigmatizantes de cônjuges, familiares, amigos e profissionais de saúde (Puhl \& Brownell, 2006; Puhl \& Heuher, 2009).

As imagens negativas que os indivíduos com excesso de peso assumem para si trazem sentimentos de inferioridade, fragilidade ou inadequação frente à sociedade (Brewis, 2014). Os problemas oriundos envolvem baixa autoestima, preocupação com o corpo, comportamentos depressivos, transtornos do comportamento alimentar, entre outras consequências de caráter social (Puhl \& Heuher, 2009). Em decorrência da estigmatização, os indivíduos podem internalizar o estigma ao reconhecer os preconceitos associados a sua condição pela sociedade e aplicar essas atitudes e crenças negativas a si, ao se tornarem conscientes dos estereótipos negativos associados a sua condição de saúde (Corrigan \&Watson, 2002; Durso \& Latner, 2008).

A experiência da estigmatização pode indicar que a desvalorização externa interfere na autoavaliação interna, ou seja, o conceito que os estigmatizados possuem sobre si pode advir da maneira como se percebem ou são vistos por outros. Eles deixam de assimilar características positivas que lhes dizem respeito e passam a deter uma atenção exacerbada ao peso, mantendo a autoavaliação negativa (Ratcliffe \& Ellison, 2013). No momento em que a pessoa com obesidade considera-se acima do peso e reconhece que a condição é desvalorizada no meio social, passa a considerar essa característica como principal aspecto do seu autoconceito (Schafer \& Ferraro, 2011). Essa situação pode envolver crenças negativas, desesperança, desequilíbrio emocional, isolamento social, desvalorização e estratégias de enfrentamento (Sikorski, Luppa, Luck, \& Riedel-Heller, 2015). As consequências influenciam, portanto, em comportamentos que levam ao ganho de peso, visto que essas pessoas são desmotivadas, possuem medo do que os outros pensam ou julgam e evitam situações que podem incidir em estigmatização (Brewis, 2014; McGuigan \&Wilkinson, 2015). Assim, os efeitos negativos à saúde física e mental associados à obesidade podem ser causados pela estigmatização relacionada ao peso e não pelo excesso de peso unicamente (Wu \& Berry, 2017).

A compulsão alimentar pode ser um comportamento de enfrentamento para lidar com o estresse causado pelo estigma de peso (Tomiyama, 2014). Os julgamentos podem levar à culpa por não possuir um corpo magro, medo, vergonha, baixa autoestima e baixa qualidade de vida, surtindo como um grande problema de saúde pública que desencadeia sofrimento nos grupos suscetíveis (Puhl \& Heuer, 2010; Tomiyama, 2014). Diante da importância do tratamento da obesidade, é necessário reduzir os preconceitos dos profissionais de saúde, aumentar a capacidade de empregar a mudança de comportamento e ampliar a colaboração de equipes multiprofissionais (Dietz et al., 2015). As consequências nocivas do estigma do peso, a estigmatização realizada pelos profissionais de saúde e a dificuldade na terapêutica da obesidade tornam a busca na literatura científica um recurso imprescindível para subsidiar a abordagem da saúde para além do corpo físico. Assim, esta pesquisa tem por objetivo realizar uma revisão integrativa da literatura sobre as possíveis consequências psicossociais do estigma do peso em adultos e sua influência no tratamento da obesidade.

\section{Metodologia}

Este artigo se trata de uma revisão integrativa que abrange as etapas de delimitação do tema e definição do procedimento: obtenção de descritores, palavras-chave, critérios de inclusão e exclusão das produções científicas; informações a serem extraídas; análise e interpretação dos resultados, discussão e síntese; e conclusão (Mendes, Silveira, \& Galvão, 2008). Foram selecionados artigos indexados nas 
bases de dados PubMed, Web of Science e PsycINFO nos últimos cinco anos (2012-2017).

Para a busca na base de dados PubMed foram utilizadas combinações de descritores do Medical Subject Headings (MeSH) e operadores boolianos OR ou AND; e para as bases de dados Web of Science e PsycINFO foram utilizados os mesmos termos, porém como palavras-chave (Quadro 1).

\section{Quadro 1}

Estratégias de busca nas bases de dados.

\begin{tabular}{|c|c|}
\hline $\begin{array}{l}\text { Base de } \\
\text { Dados }\end{array}$ & Estratégia de busca \\
\hline PubMed & 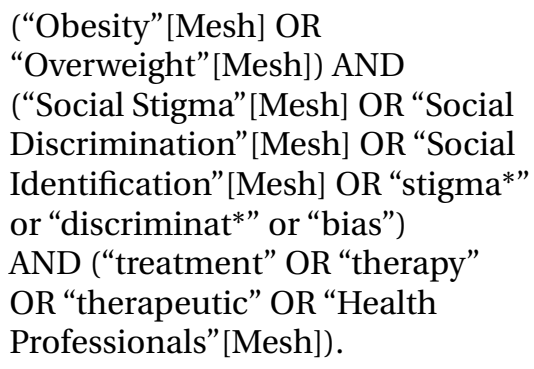 \\
\hline $\begin{array}{l}\text { Web of } \\
\text { Science e } \\
\text { PsyINFO }\end{array}$ & $\begin{array}{l}\text { (“Obesity” OR “Overweight”) } \\
\text { AND (“Social Stigma” OR “Social } \\
\text { Discrimination" OR “Social } \\
\text { Identification" OR “stigma*” } \\
\text { OR “discriminat*” OR "bias”) } \\
\text { AND (“treatment” OR "therapy” } \\
\text { OR “therapeutic” OR “Health } \\
\text { Professionals"). }\end{array}$ \\
\hline
\end{tabular}

Foram selecionados apenas artigos completos, de abordagem quantitativa ou qualitativa, experimentais ou não experimentais, com seres humanos adultos (18-65 anos), nos idiomas português e inglês. Foram incluídos aqueles que apresentavam informações que contribuem para o conhecimento das consequências do estigma do peso na efetividade do tratamento da obesidade e excluídos capítulos de livros, teses e dissertações.

Os artigos selecionados foram submetidos à leitura exploratória de textos completos, com preenchimento simultâneo à coleta de dados, constituída por título do artigo, autores e ano de publicação; objetivo da pesquisa; população do estudo; e principais resultados. Estes dados foram submetidos à análise qualitativa segundo a perspectiva bardaniana (Bardin, 2006), com interpretação e análise crítica.

\section{Resultados}

Inicialmente foram identificados os títulos dos artigos e aqueles considerados não condizentes com o objetivo deste estudo foram excluídos. Em seguida à exclusão se deu a leitura dos resumos, na qual foi identificada a presença de artigos duplicados. Os artigos considerados pertinentes para a análise $(n=15)$ foram separados para leitura completa, conforme indicado na Figura 1.

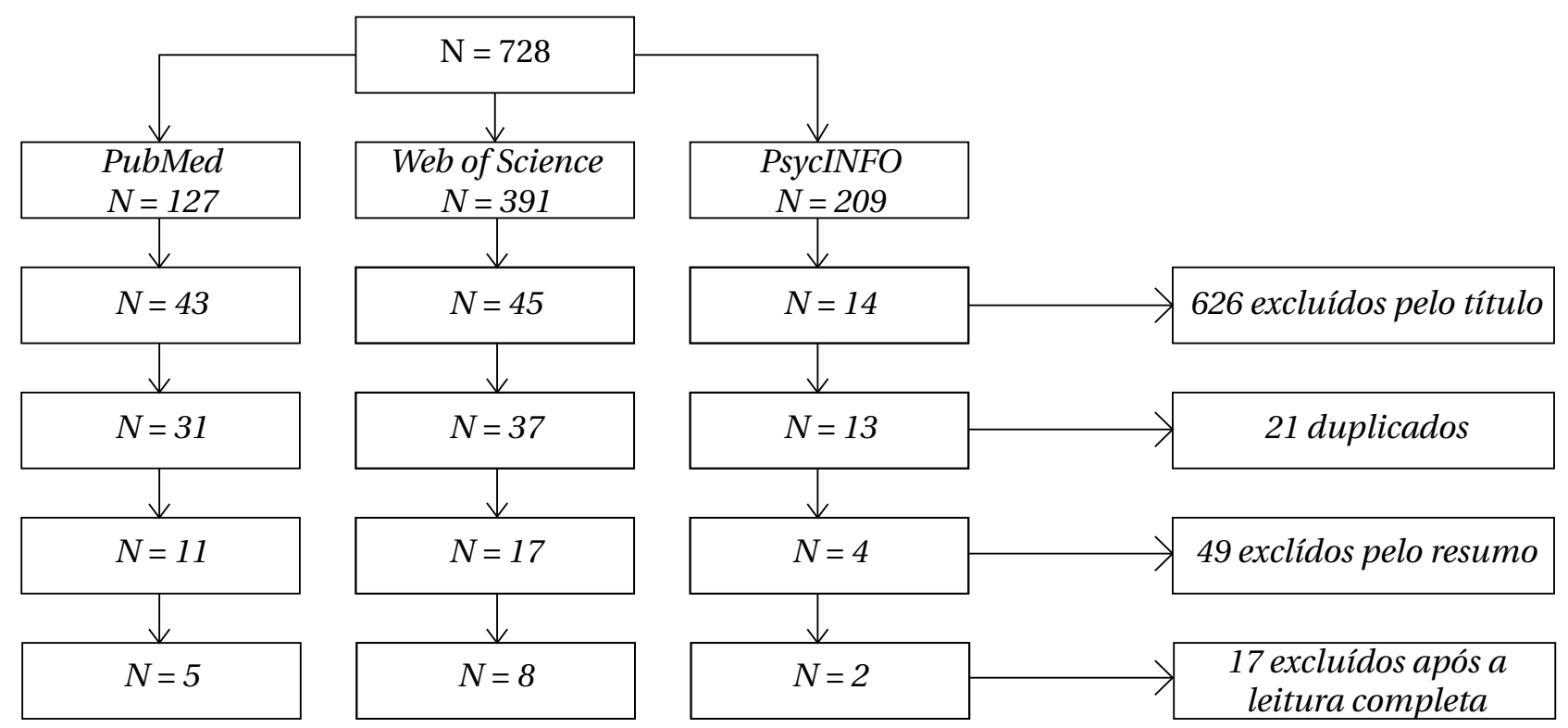

Figura 1

Esquematização do procedimento de coleta de dados da revisão integrativa. 
Dentre os artigos selecionados, catorze foram publicados em inglês e apenas um em português. Foram identificados dois subtemas: "A relação entre estigma, psicopatologias e transtornos do comportamento alimentar" e "A estigmatização entre os profissionais de saúde", conforme apresentado no Quadro 2.

\section{Quadro 2}

Caracterização dos artigos selecionados segundo autores/ano da publicação, idioma/local do estudo, objetivo e principais resultados. Ribeirão Preto - SP, 2017.

\begin{tabular}{|c|c|c|}
\hline Autores/ano & $\begin{array}{l}\text { Idioma/Local do } \\
\text { estudo }\end{array}$ & Objetivo do estudo \\
\hline $\begin{array}{l}\text { Pearl, White e } \\
\text { Grilo (2014) }\end{array}$ & $\begin{array}{l}\text { Inglês/Estados } \\
\text { Unidos }\end{array}$ & $\begin{array}{l}\text { Avaliar o impacto } \\
\text { da autoestima e da } \\
\text { sobreavaliação do corpo e } \\
\text { do peso na internalização do } \\
\text { estigma do peso em pacientes } \\
\text { com Transtorno da Compulsão } \\
\text { Alimentar Periódica (TCAP). }\end{array}$ \\
\hline
\end{tabular}

Principais resultados

O estigma internalizado foi significativamente correlacionado com a autoestima e a sobrevalorização, sendo as duas últimas significativamente correlacionadas.

$\begin{array}{ll}\text { Sutin, } & \text { Inglês/Estados } \\ \text { Robinson, Daly } & \text { Unidos } \\ \text { e Terraciano } & \\ \text { (2016) } & \end{array}$

Murakami e Inglês/Estados Latner (2015) Unidos

O'Brien et al. Inglês/Austrália (2016)

Araiza e Inglês/Estados Wellman (2017)

\section{Unidos}

Avaliar a associação entre discriminação do peso, comportamento alimentar e Índice de Massa Corporal (IMC).

Analisar a associação entre discriminação e comportamento alimentar relativas ao peso ou a discriminações por outras causas.

Avaliar as experiências estigmatizantes e a percepção de autoestima e de psicopatologia de indivíduos eutróficos e com excesso de peso que aceitavam ou não o seu peso.

Estudar a relação entre estigma do peso, internalização do estigma, estresse e transtornos do comportamento alimentar em indivíduos com diferentes classificações do estado nutricional.

Examinar a relação entre a percepção do estigma do peso e a diminuição do controle da ingestão alimentar; e verificar se essa diminuição poderia mediar a relação entre estigma do peso e a seleção de alimentos. sua condição.
A discriminação do peso foi associada ao consumo alimentar menos regular em horários e frequência e a um maior consumo de fast foods e bebidas açucaradas. A discriminação por outras causas foi associada a consumo excessivo e padrões alimentares menos regulares.

Aqueles que não aceitavam seu peso apresentaram maior estigmatização, maior psicopatologia e menor força de vontade do que aqueles que aceitavam

Os participantes com excesso de peso revelaram significativamente maior nível de estigma do peso, internalização do estigma, comer emocional e diminuição no controle do consumo alimentar.

Houve relação significativa entre percepção do estigma do peso e diminuição do controle das calorias consumidas. Contudo, o controle da ingestão alimentar não mediou a relação entre percepção do estigma do peso e a seleção de alimentos de forma significativa. 
...continuação

\begin{tabular}{|c|c|c|c|}
\hline Autores/ano & $\begin{array}{l}\text { Idioma/Local do } \\
\text { estudo }\end{array}$ & Objetivo do estudo & Principais resultados \\
\hline $\begin{array}{l}\text { Taylor, Forhan, } \\
\text { Vigod, McIntyre } \\
\text { e Morrison } \\
(2013)\end{array}$ & Inglês/Canadá & $\begin{array}{l}\text { Apresentar um quadro geral } \\
\text { sobre os efeitos da obesidade } \\
\text { na qualidade de vida - nos } \\
\text { âmbitos da saúde mental, } \\
\text { física, e social. }\end{array}$ & $\begin{array}{l}\text { A obesidade encontra-se associada } \\
\text { à maior risco de diagnóstico } \\
\text { psiquiátrico. A doença mental pode } \\
\text { dificultar a perda de peso e propagar } \\
\text { o ganho devido a hábitos alimentares } \\
\text { insalubres e comportamento } \\
\text { depressivo. }\end{array}$ \\
\hline
\end{tabular}

Puhl, Latner, King e Luedicke (2014)

Hübner et al. (2016)

Pearl et al. (2017)

Durso, Latner e Hayashi (2012)

Jung, LuckSikorski, König e Riedel-Heller (2016)
Inglês/Estados Unidos

Avaliar a estigmatização relacionada ao peso por profissionais da saúde mental especializados no tratamento de transtornos alimentares.

Inglês/Alemanha

Investigar a influência do preconceito relacionado ao peso vivenciado durante a infância e adolescência na manutenção da perda de peso durante a fase adulta e comportamentos alimentares inadequados como possíveis mediadores desta relação.

Avaliar a relação entre estigma internalizado do peso e síndrome metabólica; explorar as relações do primeiro com os fatores de risco cardiometabólicos individuais para a síndrome metabólica.

Inglês/Estados Unidos

Inglês/Alemanha

Examinar o efeito do estigma do peso e do conhecimento sobre a recomendação de cirurgia bariátrica entre médicos.
Aqueles participantes que exibiram maior estigma relacionado ao peso apresentaram a crença de que pacientes com obesidade possuem falta de vontade e incapacidade em seguir as recomendações propostas.

O preconceito relacionado ao peso durante a infância e adolescência culminou em menor efetividade na manutenção da perda de peso de adultos ao longo de dois anos de follow-up. $\mathrm{O}$ preconceito relacionado ao peso durante a infância e adolescência foi associado ao comer emocional.

A síndrome metabólica foi significativamente associada a níveis mais altos de estigma internalizado. No entanto, o único componente individual da síndrome metabólica que foi associado de forma significativa com o estigma internalizado foi a elevação de triglicerídeos.

A estigmatização foi associada a comportamentos bulímicos, compulsão alimentar e comer emocional. O estigma do peso representou um mediador parcial da relação entre discriminação e transtornos do comportamento alimentar.

58,3\% dos participantes declararam que a falta de vontade consiste no principal fator responsável pelo excesso de peso. As cirurgias bariátricas foram recomendadas mediante à perda de peso que os médicos acreditavam que poderia ser atingida. 
...continuação

\begin{tabular}{|c|c|c|c|}
\hline Autores/ano & $\begin{array}{l}\text { Idioma/Local do } \\
\text { estudo }\end{array}$ & Objetivo do estudo & Principais resultados \\
\hline $\begin{array}{l}\text { Richard, } \\
\text { Ferguson, Lara, } \\
\text { Leonard e } \\
\text { Younis (2014) }\end{array}$ & $\begin{array}{l}\text { Inglês/Estados } \\
\text { Unidos }\end{array}$ & $\begin{array}{l}\text { Examinar as variações na } \\
\text { comunicação médico- } \\
\text { paciente em pacientes com } \\
\text { diferentes classificações do } \\
\text { estado nutricional de um } \\
\text { serviço de atenção primária à } \\
\text { saúde. }\end{array}$ & $\begin{array}{l}\text { Os resultados demonstraram } \\
\text { redução na comunicação médico- } \\
\text { paciente, na possibilidade de escuta, } \\
\text { na demonstração de respeito e no } \\
\text { tempo das consultas de pessoas com } \\
\text { obesidade em comparação com outros } \\
\text { grupos. }\end{array}$ \\
\hline $\begin{array}{l}\text { Cori, Petty e } \\
\text { Alvarenga (2015) }\end{array}$ & Português/ Brasil & $\begin{array}{l}\text { Verificar as atitudes de } \\
\text { nutricionistas em relação } \\
\text { aos aspectos etiológicos da } \\
\text { obesidade e as características } \\
\text { atribuídas às pessoas com } \\
\text { excesso de peso. }\end{array}$ & $\begin{array}{l}\text { Foi observada elevada frequência } \\
\text { de respostas com atitudes } \\
\text { estigmatizantes relacionadas ao peso. } \\
\text { As pessoas com obesidade foram } \\
\text { apontadas como gulosas }(67,4 \%) \text {, } \\
\text { não atraentes }(52 \%) \text {, desajeitadas } \\
\text { (55,1\%), preguiçosas ( } 42,3 \%) \text { e } \\
\text { sem determinação }(43,6 \%) \text { pelos } \\
\text { profissionais. }\end{array}$ \\
\hline $\begin{array}{l}\text { Blodorn, Major, } \\
\text { Hunger e Miller } \\
\text { (2016) }\end{array}$ & $\begin{array}{l}\text { Inglês/Estados } \\
\text { Unidos }\end{array}$ & $\begin{array}{l}\text { Verificar a influência da } \\
\text { expectativa de rejeição } \\
\text { social nos efeitos negativos } \\
\text { do estigma do peso (menor } \\
\text { autoestima, autoconsciência, } \\
\text { estresse e ansiedade) em } \\
\text { pessoas com maior peso } \\
\text { corporal. }\end{array}$ & $\begin{array}{l}\text { Houve aumento da expectativa de } \\
\text { rejeição, menor autoestima, maior } \\
\text { autoconsciência e maior estresse em } \\
\text { mulheres com excesso de peso que } \\
\text { souberam que seriam observadas por } \\
\text { possíveis pretendentes. }\end{array}$ \\
\hline $\begin{array}{l}\text { Nicholls, } \\
\text { Pilsbury, Blake } \\
\text { e Devonport } \\
\text { (2016) }\end{array}$ & $\begin{array}{l}\text { Inglês/Reino } \\
\text { Unido }\end{array}$ & $\begin{array}{l}\text { Examinar a associação entre } \\
\text { o nível de aconselhamento } \\
\text { fornecido a pessoas com } \\
\text { obesidade, a percepção } \\
\text { de fatores etiológicos da } \\
\text { obesidade e as atitudes de } \\
\text { estudantes de enfermagem. }\end{array}$ & $\begin{array}{l}\text { Não houve associação entre as } \\
\text { atitudes relacionadas à obesidade e o } \\
\text { nível de aconselhamento oferecido. Os } \\
\text { níveis de aconselhamento oferecidos, } \\
\text { em maior frequência, foram centrados } \\
\text { no estilo de vida. }\end{array}$ \\
\hline
\end{tabular}

\section{Discussão}

\section{Subtema 1: A relação entre estigma, psicopatologias e transtornos do comportamento alimentar}

Com os resultados obtidos, observa-se um amplo enfoque na relação entre estigma, psicopatologias e patologias do comportamento alimentar. Os resultados obtidos por Pearl et al. (2014) mostraram que a autoestima e a sobrevalorização do corpo e do peso conduziram para a internalização do estigma do peso entre pacientes com TCAP. O TCAP foi relacionado com a sobrevalorização, mas não se correlacionou significativamente com o estigma internalizado ou a autoestima. A falta de correlação do TCAP com a internalização do estigma foi apontada pelos autores como consequência do intervalo restrito e gravidade dos sintomas, pois a relação entre internalização do estigma e transtornos do comportamento alimentar encontra-se bem descrita no meio acadêmico (Durso \& Latner, 2008; Durso et al., 2012; King, Puhl, Luedicke, \& Peterson, 2013; O’Brien, et al., 2016; Pearl \& Puhl, 2018; Sutin et al., 2016; Wellman, Araiza, Newell, \& McCoy, 2018).

No estudo de Sutin et al. (2016), indivíduos com sobrepeso e experiências anteriores de estigmatização relacionada ao peso revelaram maior consumo alimentar do que pessoas com sobrepeso que não 
reportaram tais experiências. Esta associação foi mais fraca entre aqueles com obesidade, o que sugere que a relação entre estigmatização do peso e comportamento alimentar não ocorre exclusivamente devido ao IMC. Outros tipos de discriminação, segundo idade, ancestrais, gênero, etnia e religião foram associados ao consumo alimentar excessivo. A discriminação relacionada à deficiência física, orientação sexual, aparência e status financeiro foram relacionadas a padrões alimentares menos regulares. No estudo de Araiza e Wellman (2017), com a ausência da situação estigmatizante não houve desregulação do controle da ingestão alimentar.

De acordo com Major, Hunger, Bunyan e Miller (2014), mais importante do que o peso é a percepção que o indivíduo tem de seu corpo e as normas voltadas para o corpo magro, predominantes na cultura ocidental, as quais podem intensificar este olhar. Os achados indicam que as mulheres que consideraram-se acima do peso e foram expostas ao conteúdo estigmatizante tiveram maior consumo alimentar e menor sensação de autocontrole (Major et al., 2014). Os resultados obtidos por Blodorn et al. (2016) apontaram para maior expectativa de rejeição, menor autoestima, maior estresse e maior autoconsciência sobre o corpo entre as mulheres que sabiam que teriam seus corpos vistos por possíveis pretendentes. Desse modo, a aceitação do peso corporal pode estar associada a menor estigmatização, maior autoestima e menos problemas psicológicos (Murakami \& Latner, 2015). Como consequência psicológica do estigma do peso, Hunger, Major, Blodorn, \& Miller (2015) destacaram o impacto do medo da rejeição social oriundo das relações interpessoais em academias de ginástica, serviços de saúde e inclusive nas relações familiares, o que indica a necessidade da abordagem emocional na assistência ao indivíduo com obesidade.

A exposição ao estigma do peso ativa a ameaça de identidade social de indivíduos com obesidade, que ocorre quando o sujeito teme ser discriminado, rejeitado ou julgado por sua identidade social (Steele, Spencer, \& Aronson, 2002). Devido ao estresse em lidar com as emoções negativas causadas pela ameaça de identidade social relacionada ao peso, o mesmo engaja-se em ações de autocontrole que causam mudança na atenção e na motivação dedicadas à ação pretendida, e assim prejudicam as tentativas subsequentes de autorregulação, promovendo menor capacidade cognitiva (Blodorn et al., 2016;
Major, Eliezer, \& Rieck 2012), menor autoestima e maior autoconsciência sobre o corpo (Blodorn et al., 2016). A perda de autocontrole pode ser relacionada à baixa capacidade de regular o consumo alimentar $\mathrm{e}$ dificuldades na manutenção ponderal (Hunger et al., 2015; Major et al., 2012).

No estudo de Major et al. (2012), foi observada depleção da função cognitiva em mulheres que sabiam que sua imagem seria vista por outras pessoas. Os autores inferem que, diferentemente de outros tipos de identidades sociais, o estigma do peso pode refletir em uma identidade em que o indivíduo frequentemente outorga a si características nocivas. Outro entrave apontado foi a inexistência de normas sociais que corrompam o preconceito e a discriminação relacionados ao excesso de peso, o que o torna ainda mais lesivo, e os acometidos, ainda mais vulneráveis (Major et al., 2012).

Moraes, Almeida e Souza (2013) identificaram que a percepção dos próprios indivíduos em relação ao seu excesso de peso esteve relacionada ao desconforto físico, como dores no corpo, ao sofrimento emocional decorrente da insatisfação com a imagem corporal, ao isolamento e à discriminação em ambiente familiar. Os indivíduos afirmaram que o consumo excessivo de alimentos decorre da necessidade de buscar conforto frente situações difíceis, e a falta de motivação para mudança foi apontada como consequência da vivência de emoções negativas associadas ao estigma.

Esses sentimentos negativos gerados pela discriminação podem ter início durante a infância e adolescência (Hübner et al., 2016), causando grande impacto na qualidade de vida relacionada à insatisfação com a imagem corporal e problemas psicossociais como baixa autoestima, ansiedade e culpa. A estigmatização no ambiente familiar propicia intensos processos psicológicos que podem levar à falta de confiança, humilhação e à falta de apoio familiar (Mattos, Perfeito, Carvalho, \& Retondar, 2012; Wilhelm, Lima, \& Schirmer, 2007). Alguns estudos têm mostrado que uma história de preconceito relacionado ao peso na infância pode estar associada com compulsão alimentar, preocupações alimentares e comportamentos compensatórios e distorcidos ao longo da vida adulta (Benas \& Gibb, 2008; Quick, McWilliams, \& Byrd-Bredbenner, 2013).

Em estudos com indivíduos que sofreram experiências de estigmatização e estavam em algum 
tratamento para perda de peso se notou a presença de compulsão alimentar (Durso et al., 2012; Puhl \& Brownell, 2006), comer emocional (Durso \& Latner, 2008; Durso et al., 2012), comportamentos bulímicos (Durso et al., 2012), diminuição da frequência das refeições (Sutin et al., 2016) e consumo de alimentos e bebidas calóricos (Araiza \& Wellman, 2017; Sutin et al., 2016).

No estudo de Sutin et al. (2016), a relação entre discriminação, consumo alimentar excessivo e consumo de fast foods foi menor em participantes com obesidade - diferentemente de outros estudos, que verificaram maior consumo calórico entre os indivíduos estigmatizados com excesso de peso, e em que mulheres com excesso de peso expostas à estigmatização do peso tiveram aumento no consumo energético (Major et al., 2014; Schvey, Puhl, \& Brownell, 2011). Sutin et al. (2016) e Durso et al. (2012) evidenciaram que o consumo excessivo surge como uma estratégia de enfrentamento da discriminação.

Com base nestes achados, observa-se que o estigma pode conduzir a uma maior dificuldade de perda de peso entre indivíduos com obesidade e contribuir para o ganho de peso ao longo do tempo (Blodorn et al., 2016). Walsh, Wadden, Tronieri, Chao e Pearl (2018) verificaram que pessoas que internalizaram o estigma do peso e estavam em tratamento para perda de peso apresentaram menor qualidade de vida independentemente do peso ou de comportamento depressivo. Além disso, manifestaram menor capacidade funcional, baixa autoestima e maior estresse, sugerindo que o tratamento da internalização do estigma do peso pode melhorar a qualidade de vida da pessoa com obesidade.

Mensinger, Tylka e Calamari (2018) observaram que pessoas com maior IMC apresentaram maior experiência e internalização do estigma do peso, e também demonstraram maior culpa e vergonha do corpo. A vergonha do corpo foi associada a estresse relacionado à assistência à saúde, que, por sua vez, foi associada à evasão dos cuidados de saúde. Desse modo, a relação entre o IMC e a evasão do serviço de saúde poderia ser explicada pelo estigma do peso experenciado ou internalizado, vergonha, culpa pelo excesso de peso e estresse, sendo necessário minimizar o estigma associado ao atendimento de profissionais de saúde com objetivo de aprimorar os serviços de prevenção, promoção e manutenção da saúde.

Ainda sobre isso, a autodepreciação com a imagem corporal é muito presente na pessoa com obesidade devido a uma grande desvalorização de sua imagem diante da visão da sociedade, o que pode resultar na manutenção da sua condição (Moraes et al., 2013). Neste aspecto, os resultados obtidos por Murakami e Latner (2015) possibilitam refletir em que medida o tratamento de perda de peso encontra-se sustentado nos pilares da autoestima e da imagem corporal, e em que medida intervenções relacionadas teriam reflexo em melhores resultados no tratamento.

\section{Subtema 2: A estigmatização entre os profissionais de saúde}

Ao avaliar a estigmatização do peso por profissionais de saúde, alguns estudos identificaram atitudes negativas em relação a pacientes com obesidade, além de certo pessimismo sobre os resultados terapêuticos (Jung et al., 2016; Puhl et al., 2014; Richard et al., 2014), sendo estes achados amplamente descritos na literatura (Cori et al., 2015; Phelan et al., 2015; Puhl \& Brownell, 2001; Puhl \& Heuer, 2009; Puhl \& Suh, 2015; Puhl et al., 2014).

A qualidade dos cuidados de saúde prestados aos indivíduos com obesidade é prejudicada devido à estigmatização relacionada ao peso. As diferenças envolvem o aconselhamento específico sobre alimentação e realização de exercício físico, modificações no estilo de vida com enfoque na perda de peso, ênfase em intervenções farmacêuticas e menor empatia nas orientações dos profissionais. A diferença no cuidado de saúde impacta na qualidade do cuidado e no abandono do tratamento (Seymour, Barnes, Schumacher, \& Vollmer, 2018).

No estudo de Puhl et al. (2014), embora os profissionais de saúde tenham referido se sentir confiantes (88\%) e preparados (84\%) para tratar pacientes com obesidade, eles afirmaram que esses indivíduos têm pouco autocontrole (33\%), são pouco atraentes (24\%), inativos (38\%), inseguros (50\%) e seu consumo alimentar é excessivo (55\%). A amostra compreendia psicólogos, nutricionistas, assistentes sociais, enfermeiros e médicos. Apenas $36 \%$ dos profissionais relataram que os pacientes com obesidade seguiam as recomendações do tratamento e somente $24 \%$ expressaram confiança na possibilidade de manutenção da perda de peso.

Os resultados de Della Torre, Courvoisier, Saldarriaga, Martin e Farpour-Lambert (2018) identificaram que $33,3 \%$ dos médicos e enfermeiros não possuíam conhecimento sobre o cálculo do IMC, além de 
relatarem falta de autoconfiança e habilidade na abordagem terapêutica de pacientes com obesidade. No estudo de Jung et al. (2016), um em cada três médicos concordaram que a perda de peso por meio da cirurgia bariátrica configura-se como um recurso que requer poucos esforços para perda de peso e, por conseguinte, foram menos propensos a recomendar a cirurgia.

Ao discutir sobre a etiologia da obesidade, os estudos selecionados verificaram que fatores causais comportamentais e emocionais receberam maior consideração do que fatores ambientais, biológicos ou genéticos (Cori et al., 2015; Puhl et al. 2014). Segundo alguns estudos, os participantes envolvidos acreditavam que pessoas com obesidade não possuem força de vontade (Brown, Stride, Psarou, Brewins, \& Thompson, 2007; Jung et al., 2016; Murakami \& Latner, 2015; Puhl, \& Heuer, 2009; 2010; Puhl et al., 2014) ou não conseguem seguir as recomendações propostas (Puhl et al., 2014). Algumas pesquisas demonstram que as explicações causais atribuídas ao controle pessoal podem exacerbar o estigma (Puhl, Schwartz, \& Brownell, 2005). Diferentemente do obtido por Richard et al. (2014), Loomis, Connolly, Clinch, \& Djuric (2001) observaram que os médicos revelaram boas atitudes para com seus pacientes, no entanto, relataram que os mesmos possuíam falta de controle.

Entre nutricionistas, de modo semelhante ao estudo de Cori et al. (2015), outros estudos encontraram características depreciativas atribuídas ao indivíduo com obesidade (Puhl \& Heuer, 2009, 2010). Berryman, Dubale, Manchester, \& Mittelstaedt (2006) avaliaram atitudes negativas em relação à obesidade entre alunos do curso de nutrição e de outros cursos da Universidade de Ohio. De modo geral, ambos os grupos exibiram níveis moderados e elevados de preconceito relacionado à gordura. A maioria dos estudantes concordou que os indivíduos com excesso de peso consomem alimentos em excesso, são inativos, lentos, inseguros, possuem baixa resistência física, baixa autoestima e baixo autocontrole. Puhl, Wharton e Heuer (2009) destacam a necessidade de intervenção na formação dos nutricionistas para aumentar a sensibilidade ao preconceito da obesidade nos currículos de nutrição, pois predomina a visão biologista da nutrição, sendo escassa a abordagem social, aumentando a probabilidade de abordagem estigmatizadora do cuidado.
Entre enfermeiros, Nicholls et al. (2016) observaram que independentemente dos fatores causais da obesidade, o tipo de aconselhamento foi o mesmo para os participantes envolvidos. Em estudo britânico com profissionais enfermeiros, um terço dos participantes afirmou que a obesidade se dá pela falta de força de vontade, e somente $8,2 \%$ mencionaram que as pessoas com obesidade são motivados à mudança de estilo de vida (Brown et al., 2007).

Castro et al. (2016) verificaram que entre discentes do curso de Nutrição e Educação física a discriminação é enfatizada nos dias atuais pela relação direta entre aparência, forma física e saúde do corpo magro/atlético. Entre os estudantes, a aparência do corpo esteve ligada a padrões estéticos cada vez mais exigentes, estabelecidos pela sociedade contemporânea. Para os estudantes de nutrição, o corpo magro foi considerado relevante para o sucesso na carreira, e entre alunos de Educação Física, observou-se que a hipertrofia do corpo significou motivo de garantia de valor e diferenciação. Segundo os autores, os estudantes desses cursos passam a exercitar o corpo para agregar status à imagem profissional, em que a preocupação com a aparência garante credibilidade; de modo que o preconceito relacionado à gordura passa a ser reforçado por estes grupos.

Do ponto de vista antropológico, David Le Breton (2006) esclarece que as ações cotidianas se dão por meio da relação do corpo com o mundo que o cerca; por essa relação o homem torna-se capaz de experenciar ações e compreender o seu entorno. Ou seja, o corpo é configurado como um agente responsável por transmitir e captar os sentidos e inserir o indivíduo em um meio social e cultural. O corpo aparece como um escopo de valores, objeto de significações, e a aparência corporal alinha-se à maneira como o indivíduo se configura em diferentes contextos.

O corpo é visto como um objeto imperfeito, e mudar de corpo passa a significar mudança de vida. O corpo idealizado não é o que possuímos, pois este deve ser modificado. Essa insatisfação com o corpo está ligada ao interesse em transformar ou eliminar o corpo que se tem. Segundo o autor, o corpo adquire uma identidade provisória, pois ainda precisa ser redefinido, modificado (Le Breton, 2003).

Congruente aos significados socialmente constituídos e inerentes ao corpo, a estigmatização é direcionada àqueles que destoam do corpo considerado adequado ou perfeito pela sociedade e está associada 
a uma pressão para a perda e controle de peso estabelecidos pelo padrão cultural vigente. Assim, o crescimento dos níveis de obesidade na sociedade contemporânea propicia o surgimento de uma sociedade que desqualifica aquele que possui excesso de tecido adiposo e que estabelece como ideal de corpo perfeito o corpo magro - o que coopera para a discriminação de indivíduos com excesso de peso (Neves \& Mendonça, 2014; Figueiredo \& Velho, 2012). Segundo os autores, a supervalorização do corpo magro menospreza o corpo da pessoa com obesidade, o que é reforçado pela falta de controle sobre este, acarretando a culpabilização do sujeito por sua condição.

As normas que definem corpos considerados adequados geram estresse nos indivíduos com obesidade, pois a mudança de hábitos pode não ser bem-sucedida, resultando em comportamento depressivo e sofrimento entre os acometidos (Taylor et al., 2013). Os indivíduos estigmatizados pelo excesso de peso passam a ter a percepção de que não são aceitos e estão distantes de obter uma relação de "iguais", o que propicia a antecipação à exclusão, à desvalorização, ao afastamento das interações sociais e à omissão da sua condição de saúde (Goffman, 1988). Dessa forma, a discriminação acompanha toda a história das pessoas com excesso de peso (Neves \& Mendonça, 2014) e é reforçada pelos processos de medicalização. Ao ser vista como "doença", a obesidade adquiriu status de epidemia, e reforça-se a ideia do "combate" por meio de medidas preventivas e terapêuticas que buscam modificar o corpo considerado defeituoso.

Como membros da sociedade, os profissionais de saúde podem ser fortemente influenciados a estigmatizar pessoas com obesidade em seu cuidado (Puhl \& Brownell, 2001; Puhl \& Heuer, 2009; Puhl et al., 2005, 2014), e, imersos nesse conceito, os mesmos deixam de oferecer a intervenção necessária, o que gera importante impacto na saúde do indivíduo - podendo complicar sua condição clínica e psíquica (Dietz et al., 2015). Os profissionais de saúde e os recursos midiáticos passaram a introduzir a crença de que as inadequações corporais são resultado da falta de cuidado sobre si (Figueiredo \& Velho, 2012).

Diante do cuidado terapêutico da obesidade, Conrad (1992) descreve que as interações sociais, históricas e fisiológicas passaram a ser vistas resumidamente como fenômenos exclusivamente biológicos, em que a medicalização da saúde prevê problemas não médicos sendo tratados como problemas médicos. A obesidade passa a ter suas consequências sociais, psicológicas e emocionais não abarcadas pelo modelo biomédico, que em detrimento à condição de patologia, passa a investir em intervenções cirúrgicas, vistas como alternativas para "corrigir" o corpo obeso e promover maior inclusão social, comparados à ineficácia promovida por intervenções de cunho comportamental propostas por meio de dietas, exercícios físicos, medicações ou tratamento psicoterápico (Neves \& Mendonça, 2014).

Além de se pensar na urbanização, as modificações dos hábitos ao longo do tempo determinadas pelo consumo excessivo, o aumento do sedentarismo e a suscetibilidade biológica e genética, e as dimensões históricas, simbólicas, psicossociais, econômicas e culturais devem ser consideradas, bem como o entendimento da obesidade em sua totalidade (Wanderley \& Ferreira, 2010). Portanto nota-se a necessidade de produção de protocolos de intervenção que atendam essas demandas e, sobretudo, gerem maior conscientização sobre o estigma do peso e suas consequências nos campos da medicina, saúde pública, nutrição e demais áreas que envolvem a obesidade.

Esta ação envolve a promoção do conhecimento sobre o estigma do peso para cientistas e profissionais de saúde envolvidos na prevenção e tratamento da obesidade (Puhl \& Suh, 2015), além da educação permanente para capacitação dos profissionais (Moraes et al., 2013). A participação de especialistas de várias áreas, em especial os profissionais de saúde mental, é essencial para que os diversos fatores associados à obesidade sejam avaliados e tratados, o que envolve a atenção às especificidades de cada pessoa pautadas em uma relação integral e humanizada, inserida nas diferentes dimensões psicossociais (Moraes et al., 2013). Além disso, empatia e compaixão permitem que cuidados de saúde voltados ao paciente sejam efetivos na otimização da relação profissional-paciente e na adesão à terapêutica (Seymour et al., 2018).

A obesidade é um problema de saúde pública, e não uma condição obtida pela negligencia de cuidados intrínsecos ao sujeito, mesmo que tais questões sejam abarcadas no trabalho multidisciplinar (Mattos et al., 2012). Algumas faculdades de medicina e centros de cirurgia bariátrica dos Estados Unidos já incorporaram a educação sobre o estigma em seu sistema para sensibilização e redução do mesmo (Puhl \& Suh, 2015). O sucesso da abordagem terapêutica depende da eficácia de sistemas e serviços para tratar 
a obesidade, de políticas públicas, de mudanças no ambiente obesogênico e do engajamento dos profissionais de saúde (Dietz et al., 2015).

Além disso, é importante que as famílias sejam orientadas sobre o diagnóstico, etiologia e impacto à saúde (Wilhelm et al., 2007). Embora possam funcionar como reforçadoras do estigma, os familiares podem auxiliar na construção de novos hábitos, pois o contexto familiar constitui vínculos que podem influenciar e modular o comportamento alimentar (Moraes et al., 2013).

\section{Considerações finais}

É possível verificar que a sociedade contemporânea encontra-se impregnada de significações que percorrem espaços socioculturais e perpetuam a figura do indivíduo com obesidade como culpado por sua condição. Este sofre e é marcado ao longo de um processo socioantropológico que deteriora o corpo obeso e o desqualifica no meio em que vive. A estigmatização da obesidade está relacionada ao comprometimento da saúde psíquica e social, cujo enfrentamento está focado no ponto de vista patológico, com estratégias de cunho comportamental que minimizam olhares para a totalidade do problema. As consequências nocivas indicam a necessidade de aumentar esforços para implementar estratégias de cuidado e atenção psicossocial específicas a esses indivíduos, além de maior sensibilização das pessoas envolvidas no contexto social, incluindo familiares e profissionais da área.

Os estudantes e profissionais de saúde parecem estar perdidos nesse confronto de ideias, afinal também sofrem com a pressão da mídia e sociedade sobre seus corpos. Sua identidade corporal reflete na visão de sucesso e exemplo a ser seguido por seus pacientes/clientes, e a perda de peso é vista como marca de sucesso do trabalho destes profissionais. Como atestam os resultados obtidos, a falta de compreensão sobre a complexidade envolvida na desvalorização do obeso e seu sofrimento agregado pode levar os profissionais a se frustrar e deixar de acreditar na causa, além de comprometer a saúde do indivíduo.

Os dados obtidos neste estudo apontam para o grande impacto psicossocial que emerge da estigmatização do peso, o que indica a necessidade de mais pesquisas que abordem esta relação e busquem intervenções mais efetivas, visto que o tratamento da obesidade promovido pelo modelo biomédico não abrange as demandas de caráter emocional e social. Inicialmente a estigmatização precisa ser visualizada como parte importante a ser abordada na terapêutica para haver sentido na busca de outros meios que atenuem a responsabilização dos sujeitos por sua condição e a visão do sujeito sobre si, amparando as questões ligadas ao seu ser.

Como limitações, é necessário destacar que a revisão integrativa de literatura compreende um processo restritivo no que diz respeito ao número de estudos incluídos para abordar o tema. No entanto pode fornecer ao leitor evidências importantes acerca do tema, enfatizando a necessidade de aumentar esforços para reduzir a estigmatização na abordagem da obesidade. Ainda assim, são necessárias pesquisas futuras que investiguem estratégias efetivas para a terapêutica da obesidade que envolvam a redução da estigmatização e o acolhimento das demandas psicossociais, visto que o estudo atual identificou dificuldades dos profissionais da saúde em lidar com a obesidade em toda sua complexidade.

\section{Referências}

Araiza, A. M., \& Wellman, J. D. (2017). Weight stigma predicts inhibitory control and food selection in response to the salience of weight discrimination. Appetite, 114, 382-390.

Bardin, L. (2006). Content analysis (L. A Rego \& A. Pinheiro, Trads.). Lisbon: Editions 70. (Trabalho original publicado em 1977)

Benas, J. S., \& Gibb, B. E. (2008). Weight-related teasing, dysfunctional cognitions, and symptoms of depression and eating disturbances. Cognitive Therapy and Research, 32(2), 143-160.

Berryman, D. E., Dubale, G. M., Manchester, D. S., \& Mittelstaedt, R. (2006). Dietetic students possess negative attitudes toward obesity similar to nondietetic students. Journal of the American Dietetic Association, 106(10), 1678-1682.

Blodorn, A., Major, B., Hunger, J., \& Miller, C. (2016). Unpacking the psychological weight of weight stigma: A rejection-expectation pathway. Journal of Experimental Social Psychology, 63, 69-76. 
Tarozo, M., \& Pessa, R. P. (2020). Impacto das Consequências Psicossociais do Estigma do Peso no Tratamento da Obesidade.

Brewis, A. A. (2014). Stigma and the perpetuation of obesity. Social Science \& Medicine, 118, 152-158.

Brewis, A. A., Wutich, A., Falletta-Cowden, A., \& Rodriguez-Soto, I. (2011). Body norms and fat stigma in global perspective. Current Anthropology, 52(2), 269-276.

Brown, I., Stride, C., Psarou, A., Brewins, L., \& Thompson, J. (2007). Management of obesity in primary care: nurses' practices, beliefs, and attitudes. Journal of Advanced Nursing, 59(4), 329-341.

Castro, J. B. P., Mattos, R. S., Passos, M. D., Aquino, F. S. D., Retondar, J. J. M., \& Machado, A. S. (2016). Alimentação, corpo e subjetividades na educação física e na nutrição: o ranço da adiposidade e a ascensão dos músculos. Demetra, 11(3), 803-824. https://orcid.org/0.12957/demetra.2016.21995

Conrad, P. (1992). Medicalization and social control. Annual Review of Sociology, 181(1), 209-232.

Cori, G. C., Petty, M. L. B., \& Alvarenga, M. S. (2015). Atitudes de nutricionistas em relação a indivíduos obesos: Um estudo exploratório. Ciência \& Saúde Coletiva, 20(2), 565-576.

Corrigan, P. W., \& Watson, A. C. (2002). The paradox of self-stigma and mental illness. Clinical Psychology-Science and Practice, 9(1), 35-53.

Della Torre, S. B., Courvoisier, D. S., Saldarriaga, A., Martin, X. E., \& Farpour-Lambert, N. J. (2018). Knowledge, attitudes, representations and declared practices of nurses and physicians about obesity in a university hospital: training is essential. Clinical Obesity, 8(2), 122-130.

Dietz, W. H., Baur, L. A., Hall, K., Puhl, R. M., Taveras, E. M., Uauy, R., \& Kopelman, P. (2015). Management of obesity: Improvement of health-care training and systems for prevention and care. The Lancet, 385(9986), 2521-2533.

Durso, L. E., \& Latner, J. D. (2008). Understanding self-directed stigma: Development of the Weight Bias Internalization Scale. Obesity, 16(S2), S80-S86.

Durso, L. E., Latner, J. D., \& Hayashi, K. (2012). Perceived discrimination is associated with binge eating in a community sample of non-overweight, overweight, and obese adults. Obes Facts, 5(6) 869-880.

Figueiredo, S. P., \& Velho, L. (2012). A medicalização da obesidade. In Jornadas Latinoamericanas de Estudios Sociales de la Ciencia y de la Tecnología, Santiago, 9, 1-28.

Goffman, E. E. (1988). Notas sobre a manipulação da identidade deteriorada. Rio de Janeiro, RJ: Guanabara.

Gracia Arnaiz, M. (2010). Alimentación y cultura en España: Una aproximación desde la antropología social. Physis: Revista de Saúde Coletiva, 20, 357-386.

Hübner, C., Baldofski, S., Crosby, R. D., Müller, A., Zwaan, M., \& Hilbert, A. (2016). Weight-related teasing and non-normative eating behaviors as predictors of weight loss maintenance. A longitudinal mediation analysis. Appetite, 102, 25-33.

Hunger, J. M., Major, B., Blodorn, A., \& Miller, C. T. (2015). Weighed down by stigma: How weight-based social identity threat contributes to weight gain and poor health. Social and Personality Psychology Compass, 9(6), 255-268.

Jung, F., Luck-Sikorski, C., König, H.H., \& Riedel-Heller, S. T. (2016). Stigma and Knowledge as Determinants of Recommendation and Referral Behavior of General Practitioners and Internists. Obes Surg, 26(10), 2393-2401.

King, K. M., Puhl, R. M. Luedicke, J., \& Peterson, J. L. (2013). Eating behaviors, victimization, and desire for supportive intervention among adolescents in weight-loss camps. Eating Behaviors, 14(4), 484-487.

Le Breton, D. (2003). Introdução: o corpo no rascunho. In: D. Le Breton, Adeus ao corpo: Antropologia e sociedade (M. Apenzeller, Trad., pp. 13-27). Campinas, SP: Papirus.

Le Breton, D. (2006). A sociologia do corpo (S. M. S. Fuhrmann, Trad.). Petrópolis, RJ: Vozes.

Loomis, G. A., Connolly, K. P., Clinch, C. R., \& Djuric, D. A. (2001). Attitudes and practices of military family physicians regarding obesity. Mil Med, 166(2), 121-125.

Major, B., Eliezer, D., \& Rieck, H. (2012). The psychological weight of weight stigma. Social Psychological and Personality Science, 3(6), 651-658.

Major, B., \& O’Brien, L. T. (2005). The social psychology of stigma. Annual Review of Psychology, 56, 393-421.

Major, B., Hunger, J. M., Bunyan, D. P., \& Miller, C. T. (2014). The ironic effects of weight stigma. Journal of Experimental Social Psychology, 51, 74-80. 
Mattos, R. S., Perfeito, P., Carvalho, M. C. V. S., \& Retondar, J. (2012). Obesidade e bullying na infância e adolescência: O estigma da gordura. Demetra, 7(2), 71-84.

McGuigan, R. D., \& Wilkinson, J. M. (2015). Obesity and healthcare avoidance: A systematic review. AIMS Public Health, 2(1), 56.

Mendes, K. D. S., Silveira, R. C. C. P., \& Galvão, C. M. (2008). Revisão integrativa: Método de pesquisa para a incorporação de evidências na saúde e na enfermagem. Texto \& Contexto - Enfermagem, 17(4), 758-764.

Mensinger, J. L., Tylka, T. L., \& Calamari, M. E. (2018). Mechanisms underlying weight status and healthcare avoidance in women: a study of weight stigma, body-related shame and guilt, and healthcare stress. Body Image, 25, 139-147.

Moraes, A. L., Almeida, E. C., \& Souza, L. B. (2013). Percepções de obesos deprimidos sobre os fatores envolvidos na manutenção da sua obesidade: investigação numa unidade do Programa Saúde da Família no município do Rio de Janeiro. Physis - Revista de Saúde Coletiva, 23(2), 553-572.

Murakami, J. M., \& Latner, J. D. (2015). Weight acceptance versus body dissatisfaction: Effects on stigma, perceived self-esteem, and perceived psychopathology. Eating Behaviors, 19, 163-167.

Neves, A. S., \& Mendonça, A. L. O. (2014). Alterações na identidade social do obeso: do estigma ao fat pride. Demetra, 9(3); 619-631. http://dx.doi.org/10.12957/demetra.2014.9461

Nicholls, W., Pilsbury, L., Blake, M., \& Devonport, T. J. (2016). The attitudes of student nurses towards obese patients: A questionnaire study exploring the association between perceived causal factors and advice giving. Nurse Education Today, 37, 33-37.

O’Brien, K. S., Latner, J. D., Puhl, R. M., Vartanian, L. R., Giles, C., Griva, K., \& Carter, A. (2016). The relationship between weight stigma and eating behavior is explained by weight bias internalization and psychological distress. Appetite, 102, 70-76.

Pearl, R. L., \& Lebowitz, M. S. (2014). Beyond personal responsibility: Effects of causal attributions for overweight and obesity on weight-related beliefs, stigma, and policy support. Psychology \& Health, 29(10), 1176-1191.

Pearl, R. L., \& Puhl, R. M. (2018). Weight bias internalization and health: A systematic review. Obesity Reviews, 19(8), 1141-1163.

Pearl, R. L., Wadden, T. A., Hopkins, C. M., Shaw, J. A., Hayes, M.R., Bakizada, Z. M., Alfaris, N., Chao, A.M., Pinkasavage, E., Berkowitz, R.I., \& Alamuddin, N. (2017). Association between weight bias internalization and metabolic syndrome among treatment-seeking individuals with obesity. Obesity, 25(2), 317-322.

Pearl, R. L., White, M. A., \& Grilo, C. M. (2014). Overvaluation of shape and weight as a mediator between self-esteem. Eating Behaviors, 15(2), 259-261.

Phelan, S. M., Burgess, D. J., Yeazel, M. W., Hellerstedt, W. L., Griffin, J. M., \& Ryn, M. (2015). Impact of weight bias and stigma on quality of care and outcomes for patients with obesity. Obesity Reviews, 16(14), 319-326.

Puhl, R. M., \& Brownell, K. D. (2001). Bias, discrimination, and obesity. Obesity Research, 9(12), 788-905.

Puhl, R. M., \& Brownell, K. D. (2006). Confronting and coping with weight stigma: an investigation of overweight and obese adults. Obesity (Silver Spring). Oct, 14(10), 1802-15.

Puhl, R. M., \& Heuer, C. A. (2009). The stigma of obesity: A review and update. Obesity, 72(6), 941-964.

Puhl, R. M., \& Heuer, C. A. (2010). Obesity stigma: Important considerations for public health. American Journal of Public Health, 100(6), 1019-1028.

Puhl, R. M., Latner, J. D., King, K. M., \& Luedicke, J. (2014). Weight bias among professionals treating eating disorders: Attitudes about treatment and perceived patient outcomes. International Journal of Eating Disorders, 47(1), 65-75.

Puhl, R. M., Quinn, D. M., Weisz, B. M., \& Suh, Y. J. (2017). The role of stigma in weight loss maintenance among US adults. Annals of Behavioral Medicine, 51(5), 754-763.

Puhl, R. M., Schwartz, M. B., \& Brownell, K. D. (2005). Impact of perceived consensus on stereotypes about obese people: A new approach for reducing bias. Health Psychology, 24, 517-552.

Puhl, R., \& Suh, Y. (2015). Health consequences of weight stigma: Implications for obesity prevention and treatment. Current Obesity Reports, 4(2), 182-190. 
Puhl, R., Wharton, C., \& Heuer, C. (2009). Weight bias among dietetics students: Implications for treatment practices. Journal of the American Dietetic Association, 438-444.

Quick, V. M., McWilliams, R., \& Byrd-Bredbenner, C. (2013). Fatty, fatty, two-by-four: Weight-teasing history and disturbed eating in young adult women. American Journal of Public Health, 103, 508-515.

Ratcliffe, D., \& Ellison, N. (2013). Obesity and internalized weight stigma: A formulation model for an emerging psychological problem. British Association for Behavioural and Cognitive Psychotherapies, 43(2), 239-252.

Richard, P., Ferguson, C., Lara, A. S., Leonard, J., \& Younis, M. (2014). Disparities in physician-patient communication by obesity status. Inquiry: The Journal of Health Care Organization, Provision, and Financing, 51, 1-7.

Schafer, M. H., \& Ferraro, K. F. (2011). The stigma of obesity: Does perceived weight discrimination affect identity and physical health? Social Psychology Quarterly, 74(1), 76-97.

Schvey, N. A., Puhl, R. M., \& Brownell, K. D. (2011). The impact of weight stigma on caloric consumption. Obesity, 19(10), 1957-1962.

Seymour, J., Barnes, J. L., Schumacher, J., \& Vollmer, R. L. (2018). A qualitative exploration of weight bias and quality of health care among health care professionals using hypothetical patient scenarios. Inquiry: The Journal of Health Care Organization, Provision, and Financing, 55, 46958018774171.

Sikorski, C., Luppa, M., Luck, T., \& Riedel-Heller S. G. (2015). Weight stigma "gets under the skin": Evidence for an adapted psychological mediation framework - a systematic review. Obesity, 23(2), 266-276.

Steele, C. M., Spencer, S. J., \& Aronson, J. (2002). Contending with group image: The psychology of stereotype and social identity threat. Advances in Experimental Social Psychology, 34, 379-440.

Sutin, A., Robinson, E., Daly M., \& Terracciano, A. (2016). Weight discrimination and unhealthy eating-related behaviors. Appetite, 102, 83-89.

Taylor, V. H., Forhan, M., Vigod, S. N., McIntyre, R. S., \& Morrison, K. M. (2013). The impact of obesity on quality of life. Best Practice \& Research Clinical Endocrinology \& Metabolism, 27, 139-146.

Tomiyama, A. J. (2014). Weight stigma is stressful: A review of evidence for the cyclic obesity/weight-based stigma model. Appetite, 82, 8-15.

Walsh, O. A., Wadden, T. A., Tronieri, J. S., Chao, A. M., \& Pearl, R. L. (2018). Weight bias internalization is negatively associated with weight-related quality of life in persons seeking weight loss. Frontiers in Psychology, 9: 2576.

Wanderley, E. N., \& Ferreira, V. A. (2010). Obesidade: Uma perspectiva plural. Ciência \& Saúde Coletiva, 15(1), 185-194.

Wellman, J. D., Araiza, A. M., Newell, E. E., \& McCoy, S. K. (2018). Weight stigma facilitates unhealthy eating and weight gain via fear of fat. Stigma and Health, 3(3), 186.

Wilhelm, F. A., Lima, J. H. C. A., \& Schirmer, K. F. (2007). Obesidade infantil e a família: Educadores emocionais e nutricionais dos filhos. Psicologia Argumento, 25(49), 143-154.

Wu, Y. K., \& Berry, D. C. (2018). Impact of weight stigma on physiological and psychological health outcomes for overweight and obese adults: A systematic review. Journal of Advanced Nursing, 74(5), 1030-1042.

\section{Maraisa Tarozo}

Nutricionista. Mestranda pelo Programa de Pós-Graduação Enfermagem em Saúde Pública da Escola de Enfermagem de Ribeirão Preto da Universidade de São Paulo, São Paulo - SP. Brasil.

E-mail: maraisatarozo@usp.br

(1) https://orcid.org/0000-0003-4146-9633

\section{Rosane Pilot Pessa}

Nutricionista. Professora associada do Departamento de Enfermagem Materno-Infantil e Saúde Pública da Escola de Enfermagem de Ribeirão Preto da Universidade de São Paulo, São Paulo - SP. Brasil.

E-mail: rosane@eerp.usp.br

(1) https://orcid.org/0000-0002-6301-6830 
Psicologia: Ciência e Profissão 2020 v. 40, e190910, 1-16.

Endereço para envio de correspondência:

Avenida dos Bandeirantes, 3.900, Campus Universitário, Monte Alegre. CEP: 14040-902. Ribeirão Preto - SP. Brasil.

Recebido 30/01/2018

Aceito 14/10/2019

Received 30/01/2018

Approved 14/10/2019

Recibido 30/01/2018

Aceptado 14/10/2019

Como citar:Tarozo, M., \& Pessa, R. P. (2020). Impacto das Consequências Psicossociais do Estigma do Peso no Tratamento da Obesidade: uma Revisão Integrativa da Literatura. Psicologia: Ciência e Profissão, 40, 1-16. https://doi.org/10.1590/1982-3703003190910

How to cite:Tarozo, M., \& Pessa, R. P. (2020). Impact of Psychosocial Consequences of Weight Stigma in the Treatment of Obesity: an Integrative Literature Review. Psicologia: Ciência e Profissão, 40, 1-16.

https://doi.org/10.1590/1982-3703003190910

Cómo citar:Tarozo, M., \& Pessa, R. P. (2020). Impacto de las Consecuencias Psicosociales del Estigma de Peso en el Tratamiento de la Obesidad: una Revisión Integrativa de la Literatura. Psicologia: Ciência e Profissão, 40, 1-16. https://doi.org/10.1590/1982-3703003190910 